\title{
A Low Cost Data Acquisition System for Measuring Shock
}

\author{
E. de Barros, A. N. Campos, F. R. Silveira, L. R. de Camargo and M. H. Mathias
}

\begin{abstract}
One of the most common environment tests is the shock testing, widely used to test the mechanical integrity and to ensure the quality and reliability of products. During shock tests, data acquisition systems are normally required for measuring acceleration time histories and processing the shock response spectrum, or SRS. This paper presents a data acquisition and analysis system based on a computer sound card for measuring shock and characterizing the shock severity by its SRS. Since the tasks of shock data acquisition frequently require expensive hardware and software, this low cost system represents a very accurate and inexpensive solution for shock characterization.
\end{abstract}

Keywords- Shock, Shock Response Spectrum, Computer Sound Card, Data Acquisition System.

\section{INTRODUÇÃO}

$\mathrm{C}$ HOQUE mecânico é definido como a excitação não periódica de um sistema, caracterizada pela sua severidade em um curto intervalo de tempo e que geralmente provoca deslocamentos relativos significantes no sistema. Se por um lado um sistema é dito exposto a um ambiente dinâmico de vibrações quando submetido a uma excitação mecânica de longa duração, por outro lado, o sistema é dito exposto ao choque quando submetido a uma entrada transiente de curta duração. Uma análise detalhada sobre choque mecânico e pirochoque é abordada na literatura [1].

Exemplos de ocorrência de choque incluem terremotos, impacto de corpos em queda livre, colisões e impactos de projéteis. Incluem também o choque de origem pirotécnica, ou pirochoque, definido como a resposta de uma estrutura a ondas de choque de altas magnitudes e altas frequências que se propagam em toda a estrutura, como resultado de um evento explosivo. As frequências dominantes em um pirochoque geralmente são maiores do que em um choque mecânico.

No que concerne à realização de testes dinâmicos necessários para a qualificação e aceitação de produtos, testes de choque são realizados em diversas áreas para verificar a resposta da estrutura às entradas transitórias a que são submetidas durante a sua vida operacional. Por exemplo, na

E. de Barros, Instituto de Aeronáutica e Espaço, São José dos Campos, SP, Brasil, everaldoeb@iae.cta.br

A. N. Campos, Instituto de Aeronáutica e Espaço, São José dos Campos, SP, Brasil, ailsonanc@iae.cta.br

F. R. Silveira, Instituto de Aeronáutica e Espaço, São José dos Campos, SP, Brasil, fabiofrs@iae.cta.br

L. R. de Camargo, Petróleo Brasileiro S.A., Exploração e Produção / Construção e Manutenção de Poços Marítimos, Santos, SP, Brasil, leandro.1rc@live.com

M. H. Mathias, Universidade Estadual Paulista "Júlio de Mesquita Filho", Guaratinguetá, SP, Brasil, mathias@feg.unesp.br
Fig. 1 é ilustrado um teste de choque realizado na área aeroespacial, para caracterizar a severidade do choque gerado durante a separação de estágios propulsores do corpo central de um foguete lançador de satélites.
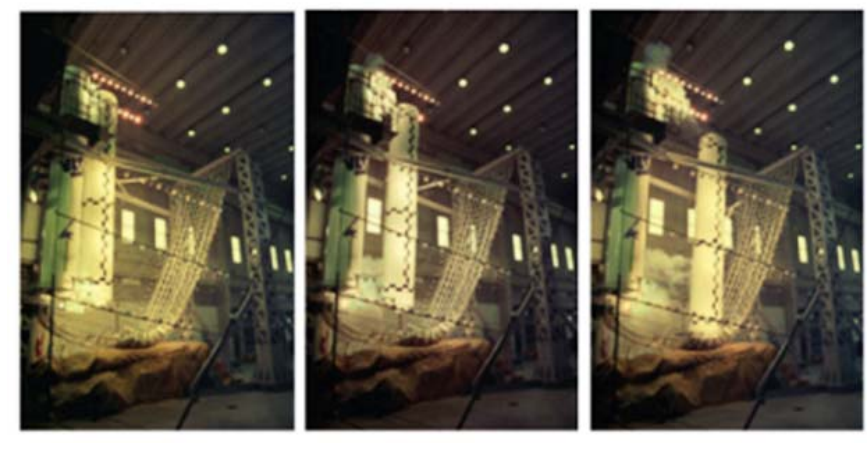

Figura 1. Execução de um teste de choque na área aeroespacial.

A Fig. 2 ilustra uma das medições de choque realizada na estrutura do estágio central do evento ilustrado na Fig. 1, representada no domínio do tempo pela sua duração e amplitude de aceleração de pico medida por um acelerômetro.

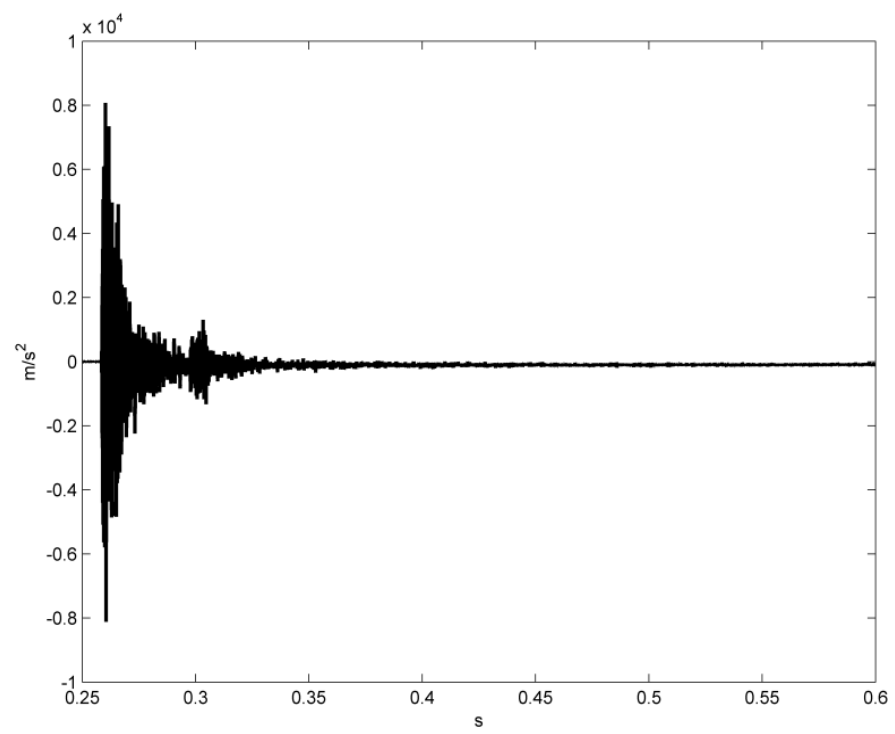

Figura 2. Medição típica de choque.

A partir de uma determinada entrada transiente, a resposta de um sistema submetido a esta entrada usualmente é analisada em termos do espectro de resposta ao choque (Shock Response Spectrum - SRS), uma representação gráfica no domínio da frequência obtida por meio da resposta máxima de um sistema de um grau de liberdade (1GDL) submetido a um pulso de entrada em sua base, variando-se a frequência natural 
do sistema $1 \mathrm{GDL}$ usualmente na faixa de $10 \mathrm{kHz}$ em intervalos de $1 / \mathrm{n}$ oitava (por exemplo 1/3, 1/6, 1/12 ou 1/24). $\mathrm{O}$ cálculo do SRS é discutido em recentes publicações [2-3].

A qualidade do espectro de SRS estimado é fortemente influenciada pela qualidade da medição do sinal de choque. A instrumentação e as técnicas empregadas na medição constituem um fator chave para garantir a qualidade dos dados amostrados e diversas implicações práticas devem ser levadas em conta na fase de medição, tais como: tipo, quantidade, faixa de frequência e faixa de amplitude dos sinais; precisão necessária para as medições; condições ambientais; tipos de análises solicitadas nos domínios do tempo e da frequência; habilidade pessoal e experiência dos analistas.

Em relação ao sistema de medição e análise, outros fatores influentes também devem ser considerados: os sistemas compreendem uma ampla variedade de preços, configurações e características, dificultando a escolha de um sistema específico para medição de choque; a relação atual com fornecedores de hardware e software e o orçamento disponível para este sistema. $\mathrm{O}$ estado da arte em termos de tais sistemas envolve as seguintes soluções: a plataforma Pulse para aquisição de dados e pós-processamento, desenvolvida pela empresa Bruel\&Kjaer; o software LMS Test.Lab Data Reduction Premium integrado com a plataforma de hardware LMS SCADAS, oferecidos pela Siemens; e ainda, o software SignalCalc Drop Test integrado com a plataforma de hardware Abacus, oferecidos pela Data Physics Corporation.

Neste contexto, sistemas de medição e análise podem apresentar elevados custos e como alternativa, a utilização da placa de som de um computador como dispositivo de aquisição de dados pode se apresentar como uma solução de baixo custo [4-10]. Este artigo apresenta um sistema de baixo custo para medição e análise de sinais de choque, baseado no emprego da placa de som de um computador para aquisição de sinais de acelerômetros e em um software desenvolvido na linguagem de programação visual Laboratory Virtual Instrument Engineering Workbench (LabView) para obtenção da representação SRS. O desempenho do sistema desenvolvido foi avaliado por meio da medição de uma entrada de choque tipo meio-seno gerada por um calibrador de choque por queda livre de esfera. Uma análise comparativa entre os valores de SRS calculados com os valores obtidos utilizando o aplicativo comercial Pulse Reflex versão 19.0.0, da empresa Bruel\&Kjaer, foi realizada para validação do sistema. Os resultados reportados sugerem que o sistema de aquisição desenvolvido representa um caminho alternativo de baixo custo para medição e análise de choque.

\section{TEORIA DA REPRESENTAÇÃO SRS}

A Fig. 3 ilustra um sistema 1GDL submetido a um pulso de entrada $u(t)$ em sua base [1]. A equação diferencial de movimento do sistema que está inicialmente em repouso é expressa por:

$-\ddot{x}(t)+2 \zeta \omega_{n} \dot{\delta}(t)+\omega_{n}^{2} \delta(t)=0$

onde $\ddot{x}(t)$ é a aceleração absoluta da massa $m$ referente ao plano inercial, $\delta(t)$ é o deslocamento relativo da massa $m$ referente ao suporte, $\omega_{n}$ é a frequência natural e $\zeta=c / 2 \sqrt{\mathrm{km}}$ é o fator de amortecimento, também expresso por $\zeta=1 / 2 Q$, onde $Q=\sqrt{\mathrm{km}} / \mathrm{c}$.

O deslocamento relativo da massa $m$ resultante do choque originado pelo pulso de entrada $u(t)$ é definido pela integral de Duhamel [1], expressa por:

$\delta(t)=\frac{1}{\omega_{d}} \int_{0}^{t} \ddot{u}(\tau) e^{-\zeta \omega_{n}(t-\tau)} \sin \omega_{d}(t-\tau) d \tau$

onde $\omega_{d}=\omega_{n} \sqrt{1-\zeta^{2}}$ é a frequência natural amortecida. A aceleração de entrada $\ddot{u}(\tau)$ é definida como uma função da variável de integração $\tau$ e a resposta $\delta(t)$ é uma função do tempo $t$. O deslocamento relativo $\delta(t)$ e a velocidade relativa $\dot{\delta}(t)$ são considerados nulos quando $t=0$. A máxima resposta que ocorre para um dado pulso de entrada $u(\tau)$ depende do valor da frequência natural amortecida $\omega_{d}$, da duração do pulso $t_{0}$, e do tempo $t$ empregado para avaliar a resposta máxima. A equação (2) indica que a resposta máxima $\delta_{\max }(t)$ é uma função de $\omega_{n}$ e $\zeta$.

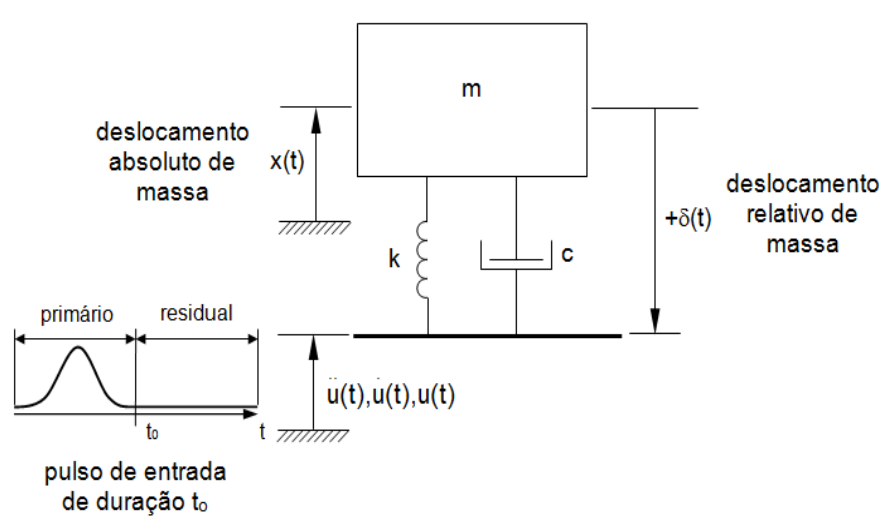

Figura 3. Modelo 1GDL submetido a uma entrada de choque na sua base.

Desta forma, o espectro SRS pode ser definido como a resposta de uma série de osciladores $1 \mathrm{GDL}$, cada um com uma frequência natural definida, submetidos a uma entrada transiente. Dados $\omega_{n}$ e $\zeta$ ou $Q$, a representação SRS é obtida por meio da resposta máxima de cada sistema 1GDL, conforme ilustrado na Fig. 4. A representação SRS obtida pelos valores de $\delta_{\max }(t)$ versus os valores de $\omega_{n}$ correspondentes para um dado fator de amortecimento, produz três tipos de espectro padronizados pela ISO 18431-4 [11]. O primeiro, quando a resposta $\delta(t)$ ao pulso de choque de entrada de duração $t_{0}$ ocorre durante o período $t<t_{0}$, a análise é denominada espectro de SRS primário. Do contrário, quando a resposta $\delta(t)$ ocorre durante o período $t>t_{0}$, o espectro é denominado SRS residual. Por fim, quando a resposta máxima é analisada em termos do valor absoluto da resposta $\delta(t)$ no período $t$, o espectro SRS é denominado maxi-max. O espectro SRS maxi-max representa a resposta mais severa possível do sistema e por este motivo é o espectro mais empregado nas análises de SRS. 


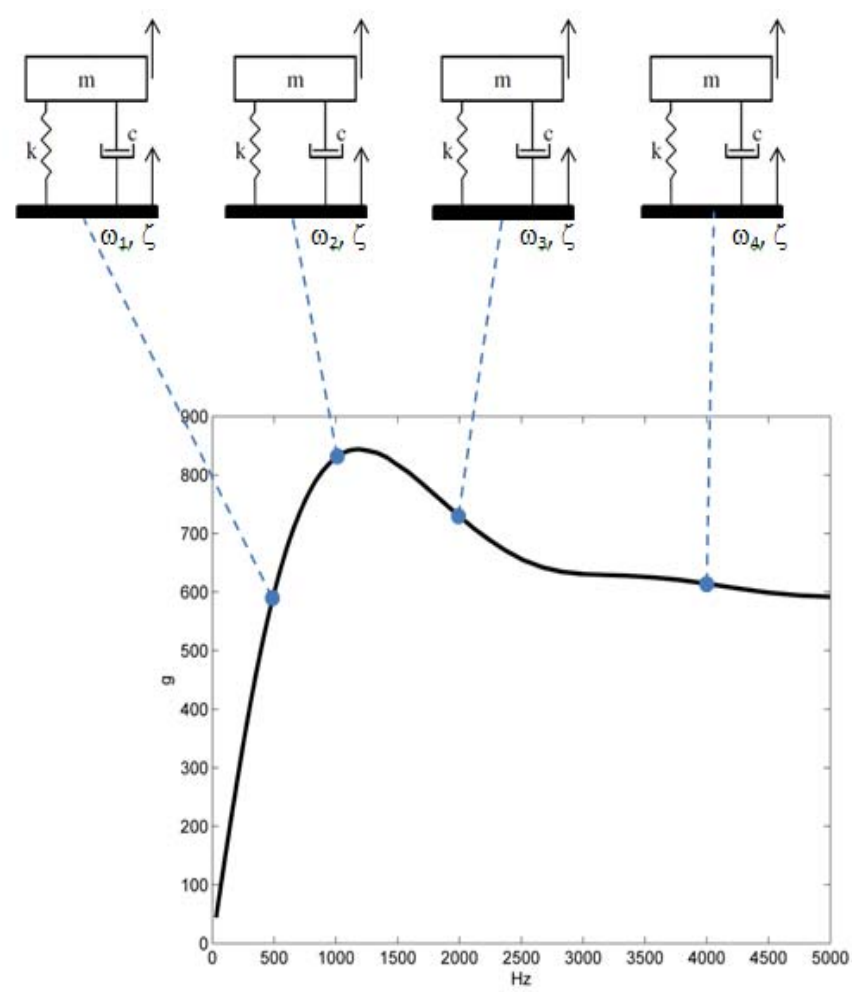

Figura 4. Representação esquemática da construção do SRS.

\section{DESCRIÇÃO DO SISTEMA DESENVOLVIDO}

Os requisitos de hardware e software para o sistema desenvolvido envolvem um notebook com sistema operacional Microsoft ${ }^{\circledR}$ Windows ${ }^{\circledR}$ com 15.6 MB de espaço livre no hard disk e 256 MB de Random Access Memory (RAM). O diagrama de fluxo do processo está ilustrado na Fig. 5.

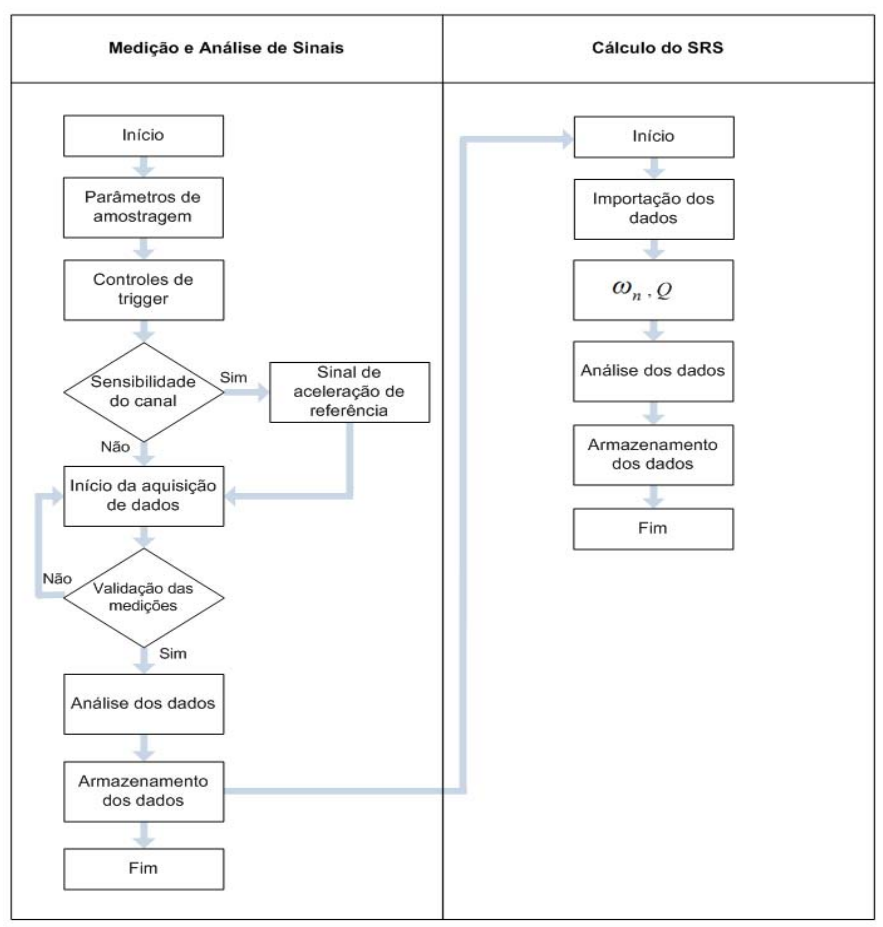

Figura 5. Diagrama de fluxo do processo.

\section{A. Módulo de medição dos sinais}

O módulo ilustrado na Fig. 6 combina as facilidades para aquisição de sinais de acelerômetros por meio dos dois canais de entrada da placa de som do computador, e posterior análise no domínio do tempo e da frequência dos sinais de vibração medidos, incluindo as seguintes funções: histórico temporal, autoespectro, densidade espectral de potência, densidade espectral cruzada, função de transferência e função de coerência. Este módulo do sistema permite ao usuário selecionar os parâmetros adequados à aquisição dos sinais, incluindo frequência de amostragem; resolução em frequência; tipo de janela temporal; número de médias; tipo de média; função de overlap; sensibilidade dos canais de entrada. Disponibiliza ainda janelas gráficas para análise do sinal selecionado, incluindo como facilidades recursos de zoom aplicados ao eixo de tempo, cursor duplo para delimitar um período específico para análise, autoescala e escala linear ou logarítmica para ambos os eixos.

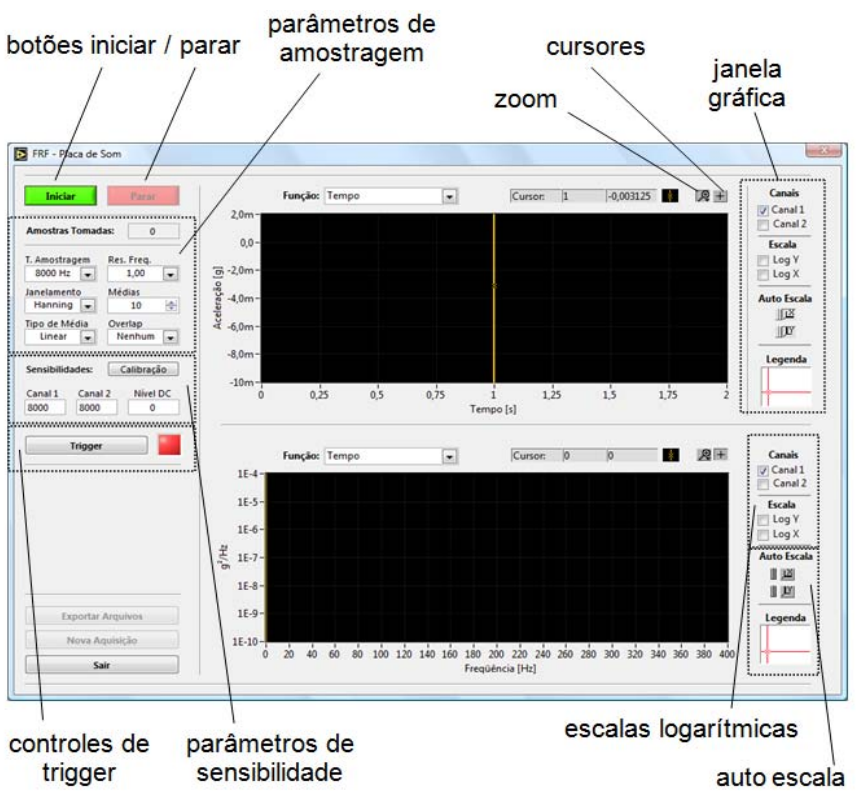

Figura 6. Módulo de medição e análise dos sinais.

A calibração de cada canal de entrada é realizada com o suporte de um padrão de referência de aceleração (por exemplo, um calibrador manual de vibração marca PCB Piezotronics modelo 394C06, que gera um nível de vibração senoidal padrão de frequência de $500 \mathrm{rad} / \mathrm{s}$ e valor global de 1 $\mathrm{g}_{\mathrm{rms}}$ ) e um acelerômetro conectado ao canal, conforme ilustrado na Fig. 7. A calibração do canal é obtida após o ajuste do valor de sensibilidade (expresso em $\mathrm{mV} /$ Unidade de Engenharia) para se atingir o valor global de aceleração gerado pelo padrão de referência.

Após a calibração dos canais de entrada e definição dos parâmetros de trigger, definida em termos de um determinado nível de aceleração em uma dada direção (positiva ou negativa) e tempo de retardo, a aquisição de dados pode ser inicializada ao se clicar no botão "Iniciar" indicado na Fig. 6, ou, do contrário, interrompida ao se clicar o botão "Parar". Quando o processo de aquisição e análise dos sinais é 
finalizado, as opções de registro e exportação dos dados permitem ao usuário eliminar dos sinais níveis DC indesejáveis, e exportar os dados das medições em formato American Standard Code for Information Interchange (ASCII) ou Universal File Format (UFF).

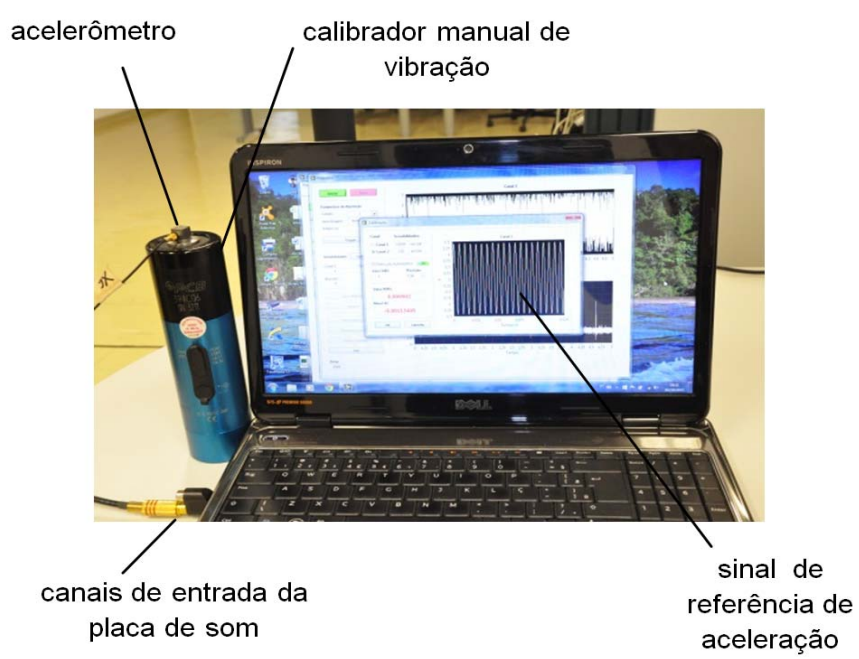

Figura 7. Vista geral do procedimento de calibração dos canais de entrada.

\section{B. Módulo de análise de SRS}

O módulo de análise de SRS ilustrado na Fig. 8 realiza a análise de SRS dos sinais de vibração medidos pelo módulo de medição e análise dos sinais. A análise é inicializada ao se clicar no botão "Iniciar" ou, do contrário, interrompida ao se clicar o botão "Parar". Para importar o histórico temporal, um botão é disponibilizado para selecionar o arquivo no formato ASCII, contendo uma coluna de tempo e outra da amplitude de vibração correspondente. A definição dos parâmetros de processamento permite ao usuário selecionar a faixa de frequência de análise, a resolução em fração de uma oitava $(1 / 3,1 / 6,1 / 12$ ou $1 / 24)$ e o fator $Q$.

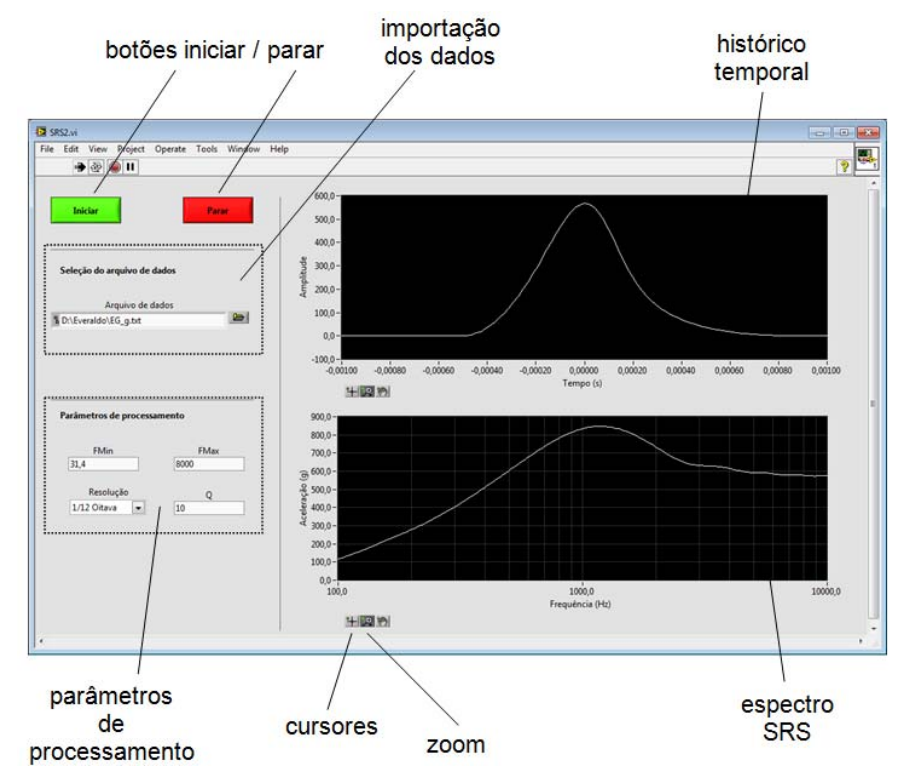

Figura 8. Módulo de análise de SRS.
Disponibiliza ainda janelas gráficas para análise do histórico temporal do sinal e o respectivo espectro SRS, incluindo como facilidades recursos de zoom aplicados aos eixos de tempo e frequência. $\mathrm{O}$ cálculo do SRS foi obtido por meio da implementação de um filtro digital de resposta ao impulso infinita (IIR) para a resposta de cada sistema 1GDL considerado no cálculo. Foi utilizado o método da invariância à rampa [12-14], cuja expressão geral da função de transferência de cada filtro IIR expressa no domínio $Z$ é dada por:

$$
H(z)=\frac{b_{20}+b_{21} z^{-1}+b_{22} z^{-2}}{1+a_{21} z^{-1}+a_{22} z^{-2}}
$$

onde $b_{20}, b_{21}, b_{22}, a_{21}$ e $a_{22}$ são os parâmetros do filtro padronizados pela ISO 18431-4, expressos por:

$$
\begin{aligned}
& b_{20}=1-\exp (-A) \frac{\sin B}{B} \\
& b_{21}=2 \exp (-A)\left(\frac{\sin B}{B}-\cos B\right) \\
& b_{22}=\exp (-2 A)-\exp (-A) \frac{\sin B}{B} \\
& a_{21}=-2 \exp (-A) \cos B \\
& a_{22}=\exp (-2 A)
\end{aligned}
$$

onde

$$
\begin{aligned}
& A=\frac{\omega_{n} T_{s}}{2 Q} \\
& B=\omega_{n} T_{s} \sqrt{1-\frac{1}{4 Q^{2}}}
\end{aligned}
$$

Desta forma, definida a taxa de amostragem $f_{s}=1 / T_{s}$ e os parâmetros $Q=1 / 2 \zeta$ e $\omega_{n}$, a função de transferência correspondente ao modelo $1 \mathrm{GDL}$ submetido a uma entrada de choque na sua base pode ser aproximada pela função discreta da equação (3).

\section{VALIDAÇÃO DO SISTEMA DESENVOLVIDO}

A validação do sistema desenvolvido foi realizada com o suporte de um calibrador de choque por queda livre de esfera marca Endevco modelo 2965C [15], ilustrado na Fig. 9. O pulso de choque do tipo meio-seno é gerado pelo calibrador da seguinte maneira: uma esfera de aço inox e de massa conhecida é posicionada no topo do calibrador. Em seguida, a esfera é solta e direcionada ao centro de uma base de rigidez 
fixada na parte inferior do calibrador. O impacto da esfera na base provoca um pulso de choque padronizado do tipo meio seno, que pode ser medido por um acelerômetro fixado nesta base.

Este sistema de calibração é utilizado para determinar a sensibilidade de acelerômetros através do choque mecânico em diferentes níveis de aceleração, variando de 20 g a 10.000 $\mathrm{g}$, com uma duração de pulso de $100 \mu \mathrm{s}$ até $3 \mathrm{~ms}$. Os valores da amplitude e a duração do pulso de choque podem ser controlados variando-se a massa da esfera, a base de rigidez ou ainda o material de interface presente na superfície da base.

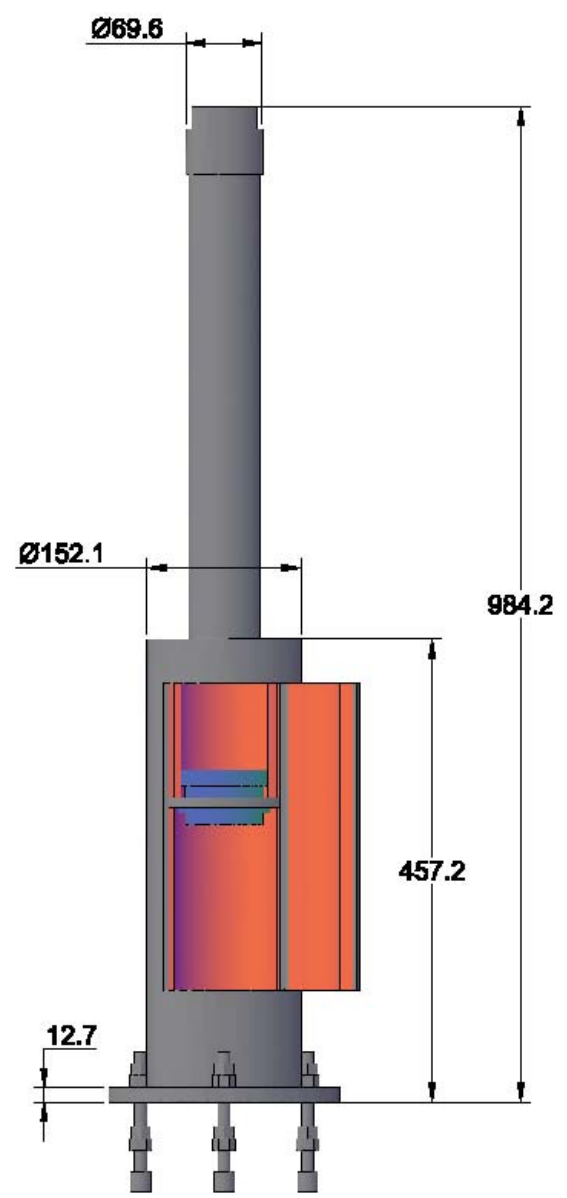

Figura 9. Calibrador de choque por queda livre de esfera.

Para o experimento ilustrado na Fig. 10, foram selecionados os seguintes acessórios Endevco para geração do choque do tipo meio-seno: uma base de rigidez código 14887 com uma esfera de aço inox com massa de $0,5345 \mathrm{~kg}$. A resposta da base de rigidez ao pulso do choque de entrada foi medido com um acelerômetro marca Brüel\&Kjaer modelo 8339 posicionado no centro da base de rigidez, e um condicionador de sinais Brüel\&Kjaer modelo 2694.

Foram utilizados os seguintes parâmetros para aquisição dos sinais: frequência de amostragem: $11.025 \mathrm{~Hz}$; resolução em frequência: $1 \mathrm{~Hz}$; janela temporal: exponencial; número de médias: 1; tipo: linear; função overlap: não aplicada. Parâmetros de configuração de trigger: nível: $10 \mathrm{~g}$; direção: positiva; tempo de retardo: $-10 \mathrm{~ms}$.

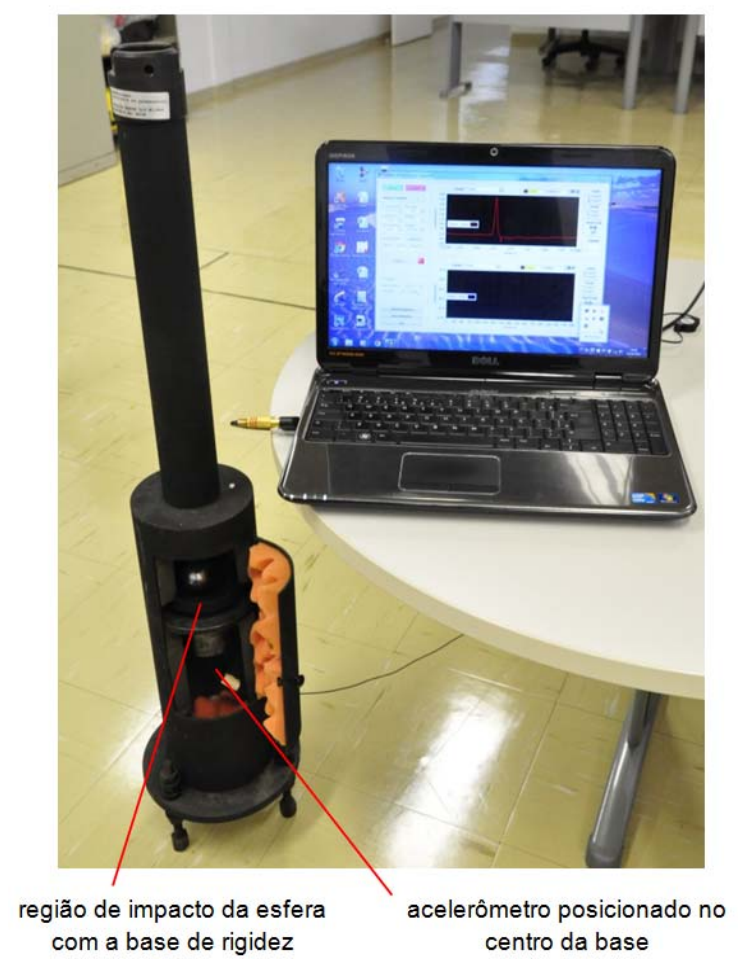

Figura 10. Vista geral do experimento.

A resposta da base de rigidez ao impacto da esfera está ilustrada na Fig. 11. Analisando esta resposta, foi observado um valor máximo de 567,5 g, que representa uma discrepância de apenas $+5,1 \%$ em relação ao valor de referência para esta combinação de acessórios do calibrador de choque. Essa discrepância é justificada pelo efeito dos componentes de incerteza presentes no resultado da medição do sistema [16].

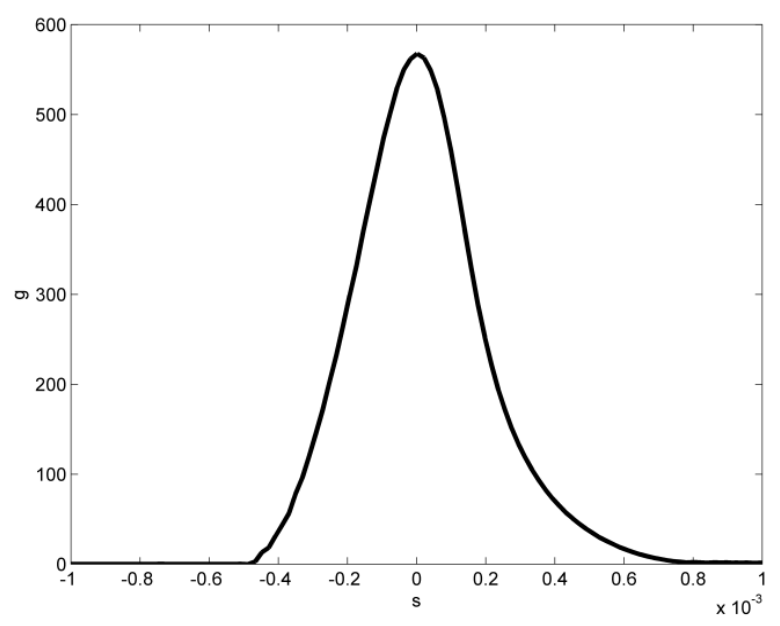

Figura 11. Medição do sinal de resposta do acelerômetro.

Após validar a medição, os valores de SRS foram obtidos para os seguintes parâmetros de processamento: faixa de frequência de análise entre $100 \mathrm{~Hz}$ e $10.000 \mathrm{~Hz}$ em intervalos de 1/12 oitava; fator $Q=10$; espectro tipo maxi-max. Em seguida, com propósitos de realização de uma análise 
comparativa entre o espectro SRS obtido pelo aplicativo desenvolvido e por um aplicativo comercial, o arquivo de dados do sinal medido foi importado no formato UFF para o aplicativo comercial Bruel\&Kjaer Pulse Reflex e os valores de SRS foram calculados para os mesmos parâmetros de processamento, conforme ilustrado na Fig. 12.

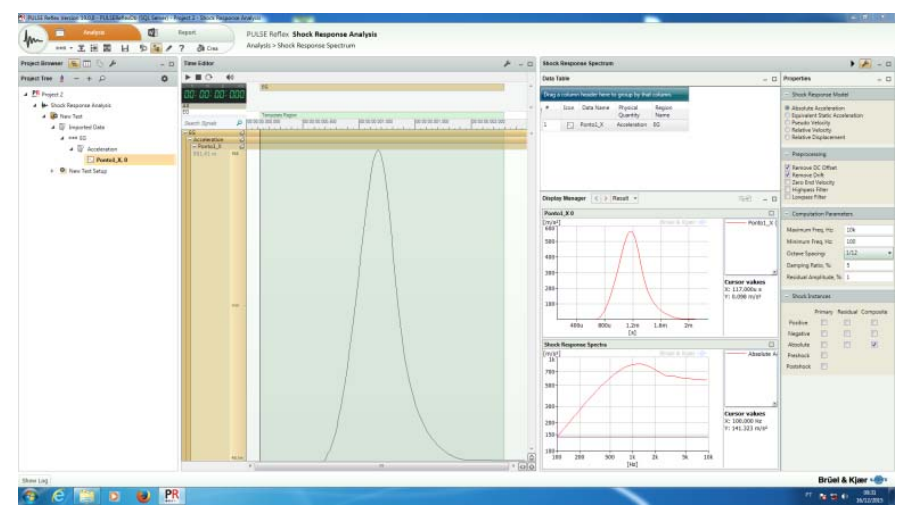

Figura 12. Análise de SRS realizada com o aplicativo comercial Pulse Reflex.

A Fig. 13 apresenta a análise comparativa dos resultados obtidos por ambos os aplicativos. Foi observada uma forte correlação entre os valores de SRS sobre uma ampla faixa de frequências, incluindo a primeira frequência natural da base de rigidez, em torno de $1.189 \mathrm{~Hz}$, evidenciando a eficiência do sistema desenvolvido.

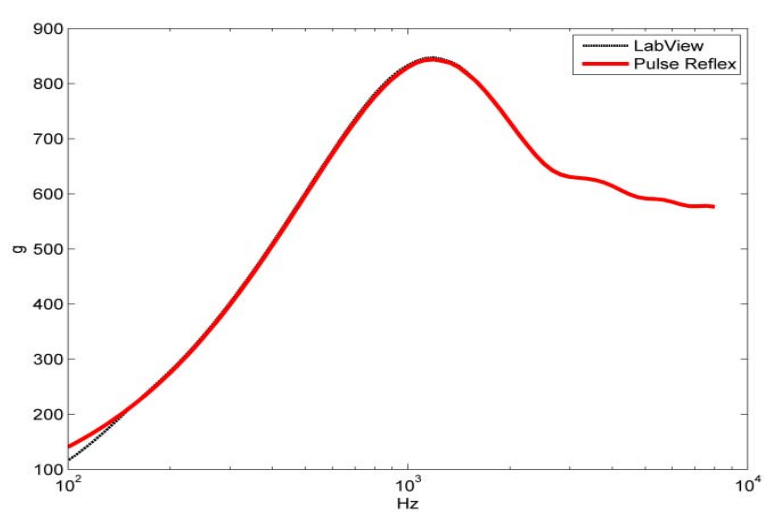

Figura 13. Análise comparativa dos espectros de SRS obtidos.

\section{CONCLUSÕES}

Em geral, toda estrutura é submetida a algum tipo de entrada transiente durante sua vida operacional que pode ser ocasionada por diferentes formas, como por exemplo, durante o transporte, o impacto de corpos em queda livre e colisões. Por este motivo, no que concerne aos requisitos para qualificação e aceitação dos produtos, entre os diversos testes recomendados e conduzidos em laboratório, são incluídos os testes de choque. A simulação de ambientes dinâmicos de choque pode ser obtida com o emprego de máquinas de choque, eventos pirotécnicos ou vibradores eletrodinâmicos. A resposta de uma estrutura quando submetida a uma entrada de choque é medida com o suporte de acelerômetros e um sistema de aquisição de dados. Usualmente, a resposta ao choque é analisada por meio do espectro SRS, uma representação gráfica obtida por meio da resposta máxima de um sistema 1GDL a uma entrada de choque. Para a medição e análise de choque, sistemas de aquisição de dados estão disponíveis em uma grande variedade de preços e desempenhos e, para uma determinada aplicação, os custos envolvidos podem se tornar proibitivos. Neste artigo foi apresentado um sistema versátil e de baixo custo para medição e análise de sinais de choque, que utiliza como hardware a placa de som de um computador para aquisição dos sinais de acelerômetros e um software desenvolvido em LabView para o processamento dos sinais e obtenção do espectro SRS. Para validação do sistema desenvolvido, foi observada a resposta ao choque gerado com o suporte de um calibrador de choque por queda livre de esfera. Os resultados experimentais obtidos foram comparados aos resultados obtidos com um aplicativo computacional comercial e indicaram uma excelente correlação entre os valores de SRS, evidenciando a eficiência do sistema.

\section{REFERÊNCIAS}

[1] C. M. Harris and A. G. Piersol, Harris' shock and vibration handbook, New York: McGraw-Hill Companies, Inc., 2002.

[2] G. Deroissart, G. Kouroussis, L. B. Fekih and O. Verlinden, "A Matlab toolbox for shock response analysis dedicated to spatial applications". In: 22nd International Congress on Sound and Vibration (ICSV22), 2015, Florence. Proceedings... Florence, 2015.

[3] T. Irvine, Vibration data pyrotechnic shock and shock response spectrum page, Vibrationdata. Disponível em: $<$ http://www.vibrationdata.com/SRS.htm>. Acesso em: 16 dez. 2015.

[4] S. Z. Simic and M. S. Kovacevic, "Computer sound card as a tool to study of fast changing electromagnetic phenomena". Computer Applications in Engineering Education, v. 21, n.1, p. 158-163, 2010.

[5] T. J. Bensky and S. E. Frey, "Computer sound card assisted measurements of the acoustic Doppler effect for accelerated and unaccelerated sound sources". American Journal of Physics, v. 69, n. 12, p. 1231-1236, 2001.

[6] A. A. Azooz, "Operating a Geiger-Müller tube using a PC sound card". European Journal of Physics, v. 30, n. 1, p. 57-67, 2009.

[7] A. A. Azooz, "Experimental demonstration of Doppler spectral broadening using the PC sound card". American Journal of Physics, v. 75, n. 2, p. 184-188, 2007.

[8] W. C. Magno, A. E. P. de Araujo, M. A. Lucena and E. Montarroyos, "Realizando experimentos didáticos com o sistema de som do PC". Revista Brasileira de Ensino de Física, v. 26, n. 2, p. 117-123, 2004.

[9] M. A. Cavalcante, A. Bonizzia and L. C. P. Gomes, "Aquisição de dados em laboratórios de física: um método simples, fácil e de baixo custo para experimentos em mecânica”. Revista Brasileira de Ensino de Física, v. 30, n. 2, 2501(1-6), 2008.

[10] E. de Barros, C. A. Souto and L. R. de Camargo, "Application of a computer sound card for measurement of mechanical vibrations". Revista Brasileira de Ensino de Física, v. 37, n. 4, 4313(1-6), 2015.

[11] ISO - International Organization for Standardization. ISO 18431-4, Mechanical vibration and shock - Signal processing - Part 4: Shock response spectrum analysis. Sep 17, 2015.

[12] D. O. Smallwood, "An improved recursive formula for calculating shock response spectra". 51st Shock and Vibration Bulletin, v. 51, n. 2, 211$217,1981$.

[13] K. Ahlin, "Comparison of test specifications and measured field data". Sound and Vibration, September, 22-24, 2006.

[14] J. Tuma, M. Babiuch and P. Koci, "Calculation of a shock response spectra". Acta Montanistica Slovaca, v. 16, n. 1, 66-73, 2011.

[15] ENDEVCO, "Specifications for model 2965C comparison shock calibrator". Manual do fabricante.

[16] A. N. Campos, Avaliação metrológica de um sistema de calibração de acelerômetros por choque mecânico, Tese de Mestrado - Curso de Engenharia Aeronáutica e Mecânica, Instituto Tecnológico de Aeronáutica, São José dos Campos - SP, 2006. 


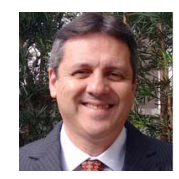

Everaldo de Barros, possui graduação em Engenharia Elétrica pela Universidade de Taubaté (1988), mestrado em Técnicas Aéronauticas e Espaciais pela École Nationale Supérieure de l'Aéronautique et de l'Espace (França,1999), mestrado em Engenharia Aeronáutica e Mecânica pelo Instituto Tecnológico de Aeronáutica (2001) e doutorado em Engenharia Mecânica pela Universidade Estadual Paulista (2004). Atualmente é tecnologista sênior do Departamento de Ciência e Tecnologia Aeroespacial. Atua como responsável pelo laboratório de vibrações mecânicas. Atua também como pesquisador do grupo de pesquisa Modelagem e Identificação de Sistemas Dinâmicos da Universidade Estadual Paulista, Campus de Guaratinguetá. Tem experiência na área de Engenharia Mecânica, com ênfase em dinâmica dos corpos rígidos, elásticos e plásticos, atuando principalmente nos seguintes temas: vibração estrutural, amortecimento, análise modal, processamento digital de sinais, instrumentação e ensaios mecânicos.

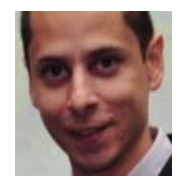

Ailson Campos Nogueira, possui graduação em Engenharia Elétrica pela Universidade do Vale do Paraíba (2000), mestrado em Engenharia Aeronáutica e Mecânica pelo Instituto Tecnológico da Aeronáutica - ITA (2010). Atualmente é tecnologista pleno do Departamento de Ciência e Tecnologia Aeroespacial. Atua como responsável pelo laboratório de instrumentação em banco de provas. Tem experiência na área de ensaios de vibração e instrumentação de ensaios com medição de pressão e força, atuando principalmente no desenvolvimento e testes de aplicações em LabVIEW destinado a ensaios.

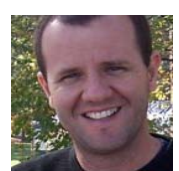

Fábio Renato Silveira, possui graduação em Engenharia Eletrica pela Universidade do Vale do Paraíba (1999), mestrando em Ciências e Técnologias Espaciais pelo Instituto Técnológico da Aeronáutica - ITA. Atualmente é professor parcial da Universidade do Vale do Paraíba ministrando aulas no curso de eletrônica. Atua como gerente técnico de laboratório de Calibração no Departamento de Ciência e Tecnologia Aeroespacial. Tem experiência na área de Engenharia Elétrica, ensaios de vibração e Metrologia.

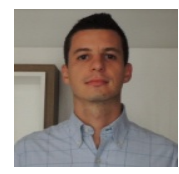

Leandro Ribeiro de Camargo, possui graduação em Engenharia Mecânica pela Universidade Estadual Paulista Júlio de Mesquita Filho (2009). Atualmente, atua como Engenheiro de Petróleo Pleno no Departamento de Construção de Poços Marítimos na Petrobras. Tem experiência na área de ensaios de vibração, análise modal e engenharia de Petróleo.

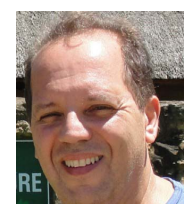

Mauro Hugo Mathias, possui graduação em Engenharia Mecânica pela Universidade Estadual Paulista Júlio de Mesquita Filho (1982), mestrado em Engenharia Mecânica pela Universidade Estadual Paulista Júlio de Mesquita Filho (1989) e doutorado em Engenharia Mecânica pela Universidade Estadual de Campinas (1998). Atualmente é professor adjunto da Universidade Estadual Paulista Júlio de Mesquita Filho, membro de grupo de trabalho de vibrações da Associação Brasileira de Ensaios não Destrutivos e Inspeção, profissional SNQC/END18538. Tem experiência na área de Engenharia Mecânica, com ênfase em Dinâmica dos Corpos Rígidos, Elásticos e Plásticos, atuando principalmente nos seguintes temas: processamento de sinais, modelagem de sistemas dinâmicos, manutenção preditiva, análise de vibrações e dinâmica de sistemas. Revisor da Revista Mechanical System and Signal Processing desde 2010. 